\title{
EFECTOS DE UN ENTRENAMIENTO CORRECTIVO EN LA ELABORACIÓN Y FUNDAMENTACIÓN DE PREGUNTAS DE INVESTIGACIÓN
}

\author{
María Antonia Padilla Vargas ${ }^{1 *}$, Natalia Fuentes González ${ }^{1}$, Virginia Pacheco Chávez ${ }^{2}$ \\ ${ }^{1}$ Centro de Estudios e Investigaciones en Comportamiento - Universidad de Guadalajara, \\ ${ }^{2}$ Facultad de Estudios Superiores Iztacala, Universidad Nacional Autónoma de México
}

Recibido, octubre 4/2014

Concepto de evaluación, marzo 28/2015

Aceptado, mayo 15/2015
Referencia: Padilla Vargas, M.A., Fuentes González, N. \& Pacheco Chávez, V. (2015). Efectos de un entrenamiento correctivo en la elaboración y fundamentación de preguntas de investigación. Acta Colombiana de Psicología, 18 (2), 87-100. DOI: 10.14718/ACP.2015.18.2.8

Resumen

\begin{abstract}
Se analizaron los efectos de un entrenamiento correctivo en la identificación y elaboración de algunos elementos de artículos empíricos (enmarcados en el Análisis Experimental de la Conducta), en el que se estudiaron los efectos de la retroalimentación y las instrucciones en tareas de discriminación condicional, con el objetivo de registrar si ello promovía el ejercicio de habilidades para elaborar y fundamentar preguntas de investigación. Participaron 20 estudiantes de psicología de una universidad pública mexicana, con edades entre los 20 y 22 años, asignados al azar a dos grupos: experimental y control. Ambos grupos fueron expuestos a una Línea Base (LB), a dos fases de entrenamiento (excepto el control) y a una sesión de evaluación. En la LB y en la evaluación los participantes debían leer dos artículos experimentales y elaborar y justificar una pregunta de investigación novedosa derivada de estos. El grupo experimental fue expuesto a un entrenamiento correctivo en la identificación (ejercicio 1) y la elaboración (ejercicio 2) de diversos elementos de artículos científicos que tenían como objetivo auspiciar desempeños en niveles intrasituacional y extrasituacional, respectivamente. Los resultados mostraron que la mayoría de los participantes del grupo experimental lograron una drástica mejoría en sus puntajes de aciertos de la LB a la evaluación, y que seis de los diez participantes fueran capaces de escribir y fundamentar sus preguntas de investigación en un nivel extrasituacional, lo que no ocurrió con los del grupo control en ningún aspecto. Los datos se discuten en términos de la importancia que tiene identificar estrategias efectivas para la formación de nuevos investigadores, sobre todo en lo relativo al desarrollo de habilidades lectoescritoras.

Palabras clave: preguntas de investigación, entrenamiento correctivo, sustitución extrasituacional, habilidades lectoescritoras, universitarios.
\end{abstract}

\section{EFFECTS OF CORRECTIVE TRAINING IN THE IDENTIFICATION AND DEVELOPMENT OF RESEARCH QUESTIONS}

\begin{abstract}
The effects of corrective training in the identification and development of some elements of empirical articles (framed within the Experimental Analysis of Behavior) were analyzed. The impact of feedback and instructions on conditional discrimination tasks were studied with the aim of determining whether this would promote the development of skills to elaborate and substantiate research questions. Twenty psychology students from a Mexican public university aged between 20 and 22 years were randomly assigned to the experimental and control groups. Both groups were exposed to a baseline, two training phases (except the control group) and to an assessment session. During baseline and assessment, participants read two experimental articles and formulated and justified a new research question derived from them. The experimental group was exposed to a corrective training in identifying (exercise 1) and developing (exercise 2) the various elements of scientific articles that were
\end{abstract}

\footnotetext{
* Centro de Estudios e Investigaciones en Comportamiento, Universidad de Guadalajara, Francisco de Quevedo \# 180, Col. Arcos Vallarta, C.P. 44130, Guadalajara, Jalisco, México. Tel: +52013338180730. tony@cencar.udg.mx. Se agradece al Programa de Mejoramiento del Profesorado (PROMEP) por los recursos para realizar el presente estudio al Cuerpo Académico: UDG-CA-53, Investigación del Comportamiento, con el apoyo a la Red temática de colaboración "Conducta humana compleja". Se agradece al Programa de Apoyo a Proyectos de Investigación e Innovación Tecnológica, DGAPA, UNAM, clave IN308213. Se agradece al Editor de la Revista, así como a los dictaminadores anónimos por sus invaluables comentarios a una versión previa del presente manuscrito, ya que éstos permitieron enriquecerlo.
} 
intended to improve reading and writing technical materials at extrasituational and intrasituational levels. Results showed that for most participants in the experimental group, correct answers increased in comparison to baseline and that six of the ten participants were able to write and justify their research questions at an extrasituational level. The control group failed on both counts. Data are discussed in terms of the importance of identifying effective strategies for training new researchers, especially regarding the development of reading and writing skills.

Key words: research questions, corrective training, extrasituational substitution, reading and writing skills, college students.

\title{
EFEITOS DE UM TREINAMENTO CORRETIVO NA ELABORAÇÃO E FUNDAMENTAÇÃO DE PERGUNTAS DE PESQUISA
}

\author{
Resumo
}

\begin{abstract}
Analisaram-se os efeitos de um treinamento corretivo na identificação e elaboração de alguns elementos de artigos empíricos (dentro da Análise Experimental da Conduta), em que se analisavam os efeitos da retroalimentação e das instruções em tarefas de discriminação condicional, com o objetivo de registrar se isso promovia o exercício de habilidades para elaborar e fundamentar perguntas de pesquisa. Participaram 20 estudantes de psicologia de uma universidade pública mexicana, com idade entre 20 e 22 anos, divididos aleatoriamente em dois grupos: experimental o controle. Ambos grupos foram expostos a uma Linha Base (LB), a duas fases de treinamento (exceto o controle) e a uma sessão de avaliação. Na LB e na avaliação os participantes deviam ler dois artigos experimentais e elaborar e justificar uma pergunta de pesquisa inovadora derivada dos mesmos. O grupo experimental foi exposto a um treinamento corretivo na identificação (exercício 1) e na elaboração (exercício 2) de diversos elementos de artigos científicos que tinham como objetivo estimular desempenhos em níveis intrasituacionais e extrasituacionais, respectivamente. Os resultados mostraram que a maioria dos participantes do grupo experimental conseguiram uma drástica melhoria em suas pontuações de acertos da LB à avaliação, e que seis dos dez participantes foram capazes de escrever e fundamentar suas perguntas de pesquisa em um nível extrasituacional, o que não aconteceu com os do grupo controle em nenhum aspecto. Os dados discutem-se em termos da importância que possuem para identificar estratégias efetivas para a formação de novos pesquisadores, sobretudo no relativo ao desenvolvimento de habilidades lecto-escritoras. Palavras chave: perguntas de pesquisa, treinamento corretivo, substituição extrasituacional, habilidades lecto-escritoras, universitários.
\end{abstract}

\section{INTRODUCCIÓN}

Formar nuevos investigadores es fundamental para el desarrollo de la ciencia. Uno de los objetivos de dicha formación es que los aprendices logren plantear preguntas de investigación novedosas (De la Fuente, Justicia, Casanova, \& Trianes, 2005; Sánchez, 2004), lo cual requiere el desarrollo de habilidades lectoescritoras que les permitan interactuar con el lenguaje técnico del campo disciplinar en el que se están formando (e.g., en psicología) (Cassany, 2006).

Se asume que los estudiantes de nivel medio y superior son capaces de leer comprensivamente, aunque varios estudios (llevados a cabo con psicólogos en formación, principalmente) han mostrado que por lo general no lo pueden hacer en niveles aceptables (Arroyo, Canales, Morales, Silva \& Carpio, 2007; Irigoyen, Jiménez \& Acuña, 2004) y su escritura es deficiente (González-Torres, Padilla \& Tamayo, 2011; Pacheco, Ramírez, Palestina \& Salazar, 2007; Padilla, Solórzano \& Pacheco, 2009; Padilla, Suro \& Tamayo, 2010; Padilla, Tamayo \& González, 2010; Tamayo, Padilla \& González-Torres, 2009).

A pesar de tal situación, al revisar los planes curriculares del nivel superior, se encuentra que el entrenamiento en habilidades lectoescritoras es más un efecto colateral que un objetivo central (Aguilera et al., 2006). Dado todo lo anteriormente expuesto, se considera necesario diseñar e implementar de manera explícita herramientas didácticas (Cassany, 2006) orientadas a dotar a los psicólogos en formación (y a todos los profesionales en general) de un entrenamiento dirigido a dominar formalmente el lenguaje de su área disciplinar, lo que implica ser capaz de leer, escribir, escuchar y hablar en los términos técnicos de la disciplina en la que se esté formando el estudiante (Moreno, 2005).

La capacidad de elaborar preguntas de investigación debidamente justificadas, como las derivadas de artículos empíricos, ha sido poco estudiada a pesar de su importancia. Dicha habilidad es fundamental para todos los investigadores, tanto para quienes se encuentran en formación, como para los ya consolidados. Elaborar preguntas de investigación implica revisar la literatura del área de interés, para poder derivarlas y fundamentarlas a partir de la evidencia empírica y teórica existente (Viniegra, 2002). Dicho proceso involucra realizar una lectura comprensiva de materiales técnicos y escribir textos en los que se debe emplear un lenguaje disciplinar.

$\mathrm{Al}$ revisar la literatura del área se encuentran muchos trabajos en los que se analiza la adquisición de la lectoescritura desde diferentes aproximaciones teóricas y con distintas estrategias (Madariaga \& Martínez, 2010; Montealegre 
\& Forero, 2006; Paradiso, 1996), pero son pocos quienes estudian las variables que influyen en el comportamiento de leer y escribir textos técnicos; de ahí la relevancia de estudiar este fenómeno tan importante para la generación y difusión del conocimiento científico.

\section{La lectoescritura desde la perspectiva interconductual}

En la teoría interconductual la lectoescritura se concibe como un campo de relaciones de interdependencia entre el individuo que lee o escribe y los otros elementos participantes en el episodio interactivo: las características del referente (lo que se lee o escribe), los criterios de logro, así como las características disposicionales de la situación, como la motivación hacia la tarea, etc. (Pacheco, 2010).

Leer y escribir pueden estructurarse como interacciones intrasituacionales, extrasituacionales y transituacionales, las cuales son progresivamente más complejas e inclusivas. Las intrasituacionales involucran interacciones que: a) implican ajustarse a la situación respondiendo a las propiedades espacio-temporales de los eventos (ej. copiar, transcribir, nombrar eventos, fenómenos o cosas); b) incluyen acciones que producen cambios en los objetos y/o eventos con los que se interactúa (ej. escribir paráfrasis de textos previamente leídos) o c) implican que el individuo se ajuste respondiendo con precisión a condiciones cambiantes en las propiedades de los objetos o sus relaciones, para producir efectos en una situación (ej. clasificar, de acuerdo con una tipificación o taxonomía, los párrafos que componen la introducción o la discusión de un artículo previamente leído) (Pacheco, 2010; Padilla et al., 2009).

El segundo tipo, denominado extrasituacional, implica interacciones en las que un participante responde a una situación en términos de las propiedades funcionales de otra. Ello posibilita relaciones desligadas de las características situacionales aparentes en los eventos, objetos $\mathrm{y} / \mathrm{o}$ organismos presentes en el ambiente; es decir, un comportamiento lingüístico (ej. plantear una pregunta de investigación novedosa debidamente justificada y derivada de artículos previamente leídos). El tercer tipo, llamado transituacional, abarca interacciones que implican un ajuste convencional ante objetos o eventos también convencionales. Las respuestas convencionales son las que relacionan o transforman dichos objetos o eventos. Estos son siempre productos lingüísticos, es decir, conceptos o símbolos (ej. elaborar una teoría como alternativa de otra existente) (Pacheco, 2010).

\section{La elaboración de preguntas de investigación}

Una actividad fundamental de la práctica científica es la elaboración de preguntas de investigación novedosas y pertinentes derivadas de un contexto teórico y empírico específico, justificadas a partir de la evidencia existente en un área concreta (Cassany, 2006; García, García, Berbén, Pichardo \& Justicia, 2014; De la Fuente, et al., 2005; Keys, Hand, Prain \& Collins, 1999; Sánchez, 2004). Proponer este tipo de preguntas demanda el ejercicio de habilidades lectoescritoras en niveles extrasituacionales, lo cual implica generar preguntas originales, y no sólo repetir lo ya realizado por otros investigadores. Pero al analizar las interacciones lectoescritoras que los estudiantes de nivel medio y superior tienen con materiales técnicos, se ha encontrado que estas suelen ocurrir únicamente en niveles intrasituacionales (Pacheco, 2010; Pacheco et al., 2007; Padilla et al., 2009).

Desde la perspectiva interconductual se ha identificado una relación directa entre el nivel funcional en el que leen y escriben estudiantes universitarios, siendo más frecuentes las ejecuciones en los niveles intrasituacionales que en los extra y transituacionales (Pacheco et al., 2007; Padilla et al., 2009). Por ejemplo, Pacheco et al. (2007) analizaron la relación entre las habilidades lectoras y de composición escrita al elaborar un anteproyecto de investigación en estudiantes de psicología. Calcularon índices de correspondencia funcional de las habilidades lectoras y escritoras, y encontraron que, excepto en un caso, tales estudiantes obtuvieron los índices de correspondencia funcional más altos en las habilidades intrasituacionales, los intermedios en las extrasituacionales y los más bajos en las transituacionales.

Posteriormente, Pacheco (2008) expuso a alumnos de licenciatura en psicología a un entrenamiento cuyo objetivo era promover que los participantes aprendieran a identificar la estructura de la introducción de un artículo empírico y a fundamentar una pregunta de investigación. El entrenamiento para el grupo experimental consistió en: 1) leer un artículo, responder preguntas relativas a este y elaborar un resumen; 2) leer dos nuevos artículos, ejemplificar, clasificar y explicar la relación entre sus párrafos; 3 ) elaborar, guiados por su profesor, la fundamentación de una pregunta de investigación propuesta por este, y 4) proponer una pregunta de investigación fundamentada en los artículos previamente leídos, y clasificar los párrafos de éstos.

Los participantes del grupo control sólo realizaban las actividades de los incisos 1 y 4, y leían los artículos del inciso 2. Los hallazgos mostraron que los participantes del grupo experimental lograron desempeños superiores a los del grupo control, y escribieron propuestas mejor estructuradas que estos.

Por otra parte, Padilla et al. (2009) analizaron los efectos de entrenar a estudiantes de posgrado para clasificar y reformular diferentes tipos de párrafos de un artículo 
experimental sobre la elaboración y fundamentación de preguntas de investigación. El entrenamiento al que se expuso a los participantes constaba de dos ejercicios diferentes: el ejercicio 1 buscaba propiciar desempeños en un nivel intrasituacional, a partir de la clasificación de los tipos de párrafos que integran un artículo experimental y la lectura de las definiciones de las variables independientes y dependientes. Por su parte, con el ejercicio 2 se esperaba propiciar desempeños en un nivel extrasituacional, mediante la identificación de cómo los párrafos que conforman la introducción de un artículo se vinculan a través de la pregunta de investigación, identificando el tipo de pregunta que se planteaba en el artículo, así como las variables manipuladas y medidas $y$, finalmente, completando y reformulando o parafraseando algunos párrafos del texto previamente leído.

El supuesto era que el entrenamiento en lectura comprensiva y en composición escrita de artículos empíricos, en los niveles intrasituacional y extrasituacional, auspiciaría la elaboración y fundamentación de preguntas de investigación en un nivel extrasituacional. Los participantes fueron expuestos a una Línea Base (LB) y a una evaluación en la que se identificó el nivel en el que elaboraban y fundamentaban una pregunta de investigación antes y después del entrenamiento. Los resultados mostraron que antes del entrenamiento todos los participantes tuvieron severas dificultades para elaborar y fundamentar sus preguntas de investigación, ya que ejercitaron los comportamientos lector y escritor en el nivel menos complejo (el intrasituacional), pero luego de exponerse a este, su desempeño mejoró cualitativamente al lograr que cuatro de los seis participantes se comportaran en niveles extrasituacionales.

El que no todos los participantes lograran desempeñarse en niveles extrasituacionales, aunado a que ninguno obtuvo el 100\% de aciertos en ninguna de las sesiones de los dos ejercicios del entrenamiento -lo cual es fundamental para lograr un desempeño óptimo en la evaluación- llevó al diseño de un nuevo experimento que implicara exponer a los participantes a un entrenamiento correctivo que los forzara a corregir los errores cometidos, promoviendo el logro del $100 \%$ de aciertos en todas las sesiones de entrenamiento. Además, se reestructuró el ejercicio 2 para que verdaderamente promoviera desempeños en niveles extrasituacionales.

El entrenamiento correctivo implica que el participante corrija, a partir de la retroalimentación respectiva, todos y cada uno de los errores cometidos antes de exponerse a una condición de prueba (es decir, sin recibir retroalimentación respecto de su desempeño). Los procedimientos de entrenamiento correctivo han sido empleados exitosamente como una estrategia didáctica que permite mejorar el desempeño de los aprendices (Oduber \& Matos, 2009; Lyster \& Ranta,
1997), así como un mecanismo que auspicia condiciones terminales equivalentes (en la fase de entrenamiento) para todos los participantes de un estudio experimental (Ribes \& Serrano, 2006; Ribes, Vargas, Luna, \& Martínez, 2009). En el presente estudio se utilizó con ambos propósitos.

Debido a todo lo anteriormente expuesto, el objetivo del presente estudio fue analizar el efecto de un entrenamiento correctivo en la identificación y elaboración de algunos de los elementos que conforman un artículo empírico, en el planteamiento y justificación de preguntas de investigación, en estudiantes de psicología.

\section{MÉTODO}

\section{Participantes}

Participaron 20 estudiantes de cuarto y quinto semestre de la carrera de psicología de una universidad pública mexicana, 17 mujeres y 3 hombres, cuyas edades fluctuaron entre los 20 y los 22 años. La participación fue voluntaria por invitación directa. En el estudio participaron más mujeres que hombres debido a que en dicha carrera suele haber mayor proporción femenina que masculina. Ninguno de los participantes tenía experiencia en este tipo de tareas experimentales, y a cambio de su participación, se les dieron créditos en una de sus asignaturas.

\section{Materiales}

Se emplearon cuatro artículos experimentales en los que se analizaban los efectos de la retroalimentación y las instrucciones en tareas de discriminación condicional. Todos los estudios fueron llevados a cabo en el marco del Análisis Experimental de la Conducta y tenían una extensión de once a doce cuartillas. Además se usaron diversos materiales diseñados ex profeso, los cuales se describen en el apartado de Procedimiento. Se eligieron tales artículos en concreto debido a que pertenecían a una misma área de conocimiento, tenían una extensión similar, pero sobre todo, debido a que cumplían con los requisitos de: a) tener claramente descrita la pregunta de investigación con su variable independiente, dependiente, y donde fuera clara su vinculación, b) que se argumentara la pertinencia de la pregunta de investigación y dichos argumentos aparecieran en el mismo párrafo que la pregunta, y c) que se expusiera detalladamente la evidencia empírica relacionada con los diferentes elementos de la pregunta de investigación correspondiente.

\section{Escenario}

Las sesiones experimentales se realizaron en cubículos sonoamortiguados de 1.0 por 1.5 metros, que contaban con 
iluminación natural y artificial. En cada cubículo había una mesa, una silla y un equipo de cómputo.

\section{Diseño}

Se formaron dos grupos al azar: uno experimental y uno control. El grupo experimental fue expuesto a una Línea Base (LB) de dos sesiones, a dos ejercicios (ejercicios 1 y 2) de entrenamiento, cada uno de dos sesiones, y a una evaluación de una sesión; mientras que el grupo control se expuso a la LB, a la lectura de los artículos empleados en los dos ejercicios con el grupo experimental, y a la evaluación. Los participantes del grupo control leían los artículos empleados con el grupo experimental debido a que en la evaluación debían derivar de estos su pregunta de investigación. Además, se quería probar si la sola lectura de tales materiales auspiciaba que los sujetos fueran capaces de elaborar y fundamentar preguntas de investigación en niveles extrasituacionales. En la Tabla 1 se presenta el diseño experimental empleado.

\section{Procedimiento}

Los participantes resolvían la tarea individualmente. Antes de iniciar, leían una carta de consentimiento informado, y si estaban de acuerdo con lo estipulado en ésta, la firmaban. Solo quienes firmaban dicho consentimiento participaban en el estudio.

1. Los sujetos del grupo experimental se exponían a siete sesiones, y los del grupo control a cuatro. Las sesiones tenían una duración aproximada de una hora, dependiendo de cada participante, y se realizaba una sesión por día. Ambos grupos debían leer dos artículos y elaborar un anteproyecto de investigación tanto en la fase de LB como en la de evaluación. La pregunta de investigación, y la correspondiente fundamentación del anteproyecto elaborado, debía derivarse de los artículos previamente leídos.

$1.2 \mathrm{El}$ grupo experimental se exponía a dos sesiones en la LB; en la primera leían un artículo y podían hacer anotaciones, si lo deseaban. En la segunda sesión leían otro texto y debían responder a las siguientes preguntas respecto del artículo leído: pregunta de investigación planteada, tipo de pregunta de éste (con base en una clasificación diseñada ex profeso), variable(s) independiente(s) y dependiente(s). Finalmente, con base en los dos artículos leídos debían elaborar un anteproyecto de investigación que contuviera (para ello se les proporcionaba una guía diseñada para tal fin), una pregunta de investigación derivada de los artículos previamente leídos, por lo menos una variable dependiente, una variable independiente, evidencia(s) que diera(n) soporte a la pregunta elaborada, argumentos que justificaran la propuesta, describir qué participantes sería conveniente emplear para responder a la pregunta elaborada, el procedimiento, los resultados esperados y explicar de qué manera creían que se vinculaban tales resultados con la pregunta de investigación propuesta. No había límite de tiempo para realizar ninguna de las actividades del estudio.

Tabla 1.

Diseño experimental empleado

\section{Grupo Experimental}

a) Leer un artículo diferente a los empleados en la LB, b) a) Leer un artículo (el mismo leído por los participantes leer un texto acerca de las preguntas de investigación, c) del grupo experimental), y b) leer un texto acerca de las responder a las mismas preguntas que en la segunda sesión preguntas de investigación. de la LB, e d) identificar algunos elementos de un artículo experimental.

Sesión 2 Similar a la sesión anterior, pero con un artículo diferente.

a) Leer el mismo artículo empleado en el Ejercicio 1, b) responder a varias preguntas respecto de cómo se vinculan los diferentes elementos del artículo entre sí, c) proponer una tarea diferente a la empleada en el artículo leído para medir las mismas variables analizadas en éste, d) sugerir el estudio de una variable no contemplada en dicho artículo, e) formular una pregunta de investigación y, f) explicar resultados alternativos con base en lo expuesto por el autor del artículo leído.

Sesión 2 Similar a la sesión anterior, pero con un artículo diferente.
Similar a la sesión anterior, pero con un artículo diferente.

Leer el mismo artículo que los participantes del grupo experimental.

Similar a la sesión anterior, pero con un artículo diferente.

Nota: Se describen las actividades a realizar en cada una de las sesiones de que constaban los ejercicios 1 y 2. 
1.2.1 En una segunda fase se exponía al grupo experimental a un entrenamiento que constaba de dos sesiones para cada uno de dos ejercicios. El ejercicio 1 (diseñado para promover desempeños en niveles intrasituacionales) consistía en leer un nuevo artículo, leer un texto acerca de las preguntas de investigación (preparado ex profeso) y responder a las mismas preguntas que se habían formulado en la segunda sesión de la LB. Además, debían identificar en qué parte del artículo había Definiciones, Evidencias, Formulación de preguntas y Objetivos, Planteamientos derivados o una combinación de dos o más de tales elementos.

Los participantes se exponían a dos sesiones con dos artículos diferentes, y al concluir cada sesión se retroalimentaba su desempeño. En cada caso debían corregir los errores cometidos. Tenían tres oportunidades para obtener la respuesta correcta, y si no lo hacían, en la tercera ocasión se les indicaba cuál era esta y se continuaba con la siguiente sesión.

1.2.2 El ejercicio 2 (diseñado para promover desempeños en niveles extrasituacionales) también constaba de dos sesiones en las que el participante debía leer los mismos artículos que en el ejercicio anterior (véase la Tabla 2 para una descripción esquemática de las actividades las actividades a realizar en cada una de las sesiones de que constaban los ejercicios 1 y 2). A partir de la lectura de cada uno de los textos debía responder a las preguntas: ¿cómo se relacionaba la introducción con la pregunta de investigación del artículo leído?, ¿cómo se justificaba la elaboración del estudio?, ¿cómo se vinculaban las evidencias presentadas en la introducción con los resultados? Además, debía proponer una tarea diferente a la empleada en el artículo leído para medir las mismas variables analizadas en este, y sugerir el estudio de una variable no contemplada en el artículo, para ser analizada. También debía formular una pregunta de investigación que incluyera la variable propuesta, explicitando variables dependientes e independientes. Finalmente, debía explicar resultados alternativos con base en lo expuesto por el autor del artículo leído.

La retroalimentación se proporcionaba de forma similar al ejercicio 1. La fase de evaluación se llevaba a cabo en una sola sesión e implicaba elaborar una pregunta de investigación y su correspondiente justificación, basándose en los textos leídos anteriormente y con la misma guía empleada en la LB. Al concluir se les proporcionaba retroalimentación respecto de su ejecución. Los participantes del grupo control leían los mismos artículos que el grupo experimental para la LB y la evaluación, pero no se les proporcionaba ningún tipo de entrenamiento ni retroalimentación.

Tabla 2.

Descripción esquemática de los Ejercicios 1 y 2 que formaban parte del entrenamiento

Grupo Experimental

Grupo Control

\begin{tabular}{|c|c|c|c|}
\hline \multirow[t]{2}{*}{ 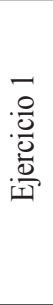 } & Sesión 1 & $\begin{array}{l}\text { a) Leer un artículo diferente a los empleados en la LB, b) } \\
\text { leer un texto acerca de las preguntas de investigación, c) } \\
\text { responder a las mismas preguntas que en la segunda sesión } \\
\text { de la LB, e d) identificar algunos elementos de un artículo } \\
\text { experimental. }\end{array}$ & $\begin{array}{l}\text { a) Leer un artículo (el mismo leído por los } \\
\text { participantes del grupo experimental), y } \\
\text { b) leer un texto acerca de las preguntas de } \\
\text { investigación. }\end{array}$ \\
\hline & Sesión 2 & Similar a la sesión anterior, pero con un artículo diferente. & $\begin{array}{l}\text { Similar a la sesión anterior, pero con un } \\
\text { artículo diferente. }\end{array}$ \\
\hline \multirow[t]{2}{*}{ 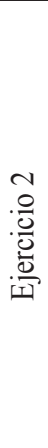 } & Sesión 1 & $\begin{array}{l}\text { Leer el mismo artículo empleado en el Ejercicio 1, b) } \\
\text { responder a varias preguntas respecto de cómo se vinculan } \\
\text { los diferentes elementos del artículo entre sí, c) proponer } \\
\text { una tarea diferente a la empleada en el artículo leído para } \\
\text { medir las mismas variables analizadas en éste, d) sugerir el } \\
\text { estudio de una variable no contemplada en dicho artículo, } \\
\text { e) formular una pregunta de investigación y, f) explicar } \\
\text { resultados alternativos con base en lo expuesto por el autor } \\
\text { del artículo leído. }\end{array}$ & $\begin{array}{l}\text { Leer el mismo artículo que los participantes } \\
\text { del grupo experimental. }\end{array}$ \\
\hline & Sesión 2 & Similar a la sesión anterior, pero con un artículo diferente. & $\begin{array}{l}\text { Similar a la sesión anterior, pero con un } \\
\text { artículo diferente. }\end{array}$ \\
\hline
\end{tabular}

Nota: Se describen las actividades a realizar en cada una de las sesiones de que constaban los ejercicios 1 y 2. 
Análisis de resultados

Con respecto al análisis de los datos, este se realizó tanto cuantitativa como cualitativamente. Para la parte cuantitativa se registró el porcentaje de respuestas correctas obtenido por cada participante en la LB, en los ejercicios 1 y 2 , y en la evaluación, empleando para ello un Baremo diseñado ex profeso por medio del cual se calificaba la claridad y pertinencia con la que los participantes explicitaban cada uno de los aspectos que conforman la elaboración y fundamentación de una pregunta de investigación (ej. pregunta de investigación, variables independientes y dependientes, argumentos empleados en la fundamentación, etc.).

Se registró también la extensión de las propuestas (número de palabras) tanto en la LB como en la evaluación, para identificar si como efecto del entrenamiento, los participantes las elaboraban de manera más detallada en la fase de evaluación. Por otro lado, para la parte cualitativa se analizó la precisión del empleo de los conceptos técnicos utilizados en la LB y en la evaluación, con el propósito de observar si existía algún efecto del entrenamiento sobre este aspecto, así como la congruencia de las propuestas elaboradas (en otro lugar de este escrito se proporcionan los criterios para su evaluación), y el nivel funcional en el que los participantes escribían sus preguntas de investigación y la justificación de estas, tanto en la LB como en la evaluación, según los siguientes criterios: una pregunta y su correspondiente justificación se consideraban escritas en un nivel intrasituacional cuando la pregunta o la justificación eran meras copias de los artículos previamente leídos o cuando no había correspondencia entre la pregunta y la justificación propuestas.
Se consideraban extrasituacionales si se proponía una manipulación que no se hubiera realizado en los artículos revisados o se modificaba el diseño, la metodología o los análisis propuestos; si esta se derivaba lógicamente de lo leído, y si había congruencia entre la pregunta de investigación y su justificación.

Dado que el criterio de dicha tarea era que se elaborara una pregunta derivada, cuando la propuesta del participante no tenía ninguna relación con los artículos leídos, esta se consideraba no pertinente y se evaluaba como ausencia de respuesta. Con el objeto de dar confiabilidad al análisis de resultados, las respuestas de los participantes fueron analizadas por dos calificadores independientes. En caso de discrepancia se discutía cada dato hasta llegar a un acuerdo, por lo que la confiabilidad fue de $100 \%$ en todos los casos.

\section{RESULTADOS}

En esta sección se muestra el porcentaje de aciertos obtenido en la Línea Base y la Evaluación por los participantes de los dos grupos: experimental y control, así como el porcentaje de respuestas correctas obtenido en las tres sesiones del entrenamiento al que se expuso el grupo experimental. Posteriormente se presentan los resultados relativos a la extensión, pertinencia, congruencia y nivel funcional del anteproyecto elaborado por los participantes de los dos grupos mencionados previamente.

En la Figura 1 se muestra el porcentaje de respuestas correctas obtenido en la LB, y la evaluación por los participantes de los grupos experimental y control. En el eje horizontal se muestra a los participantes, y en el vertical,
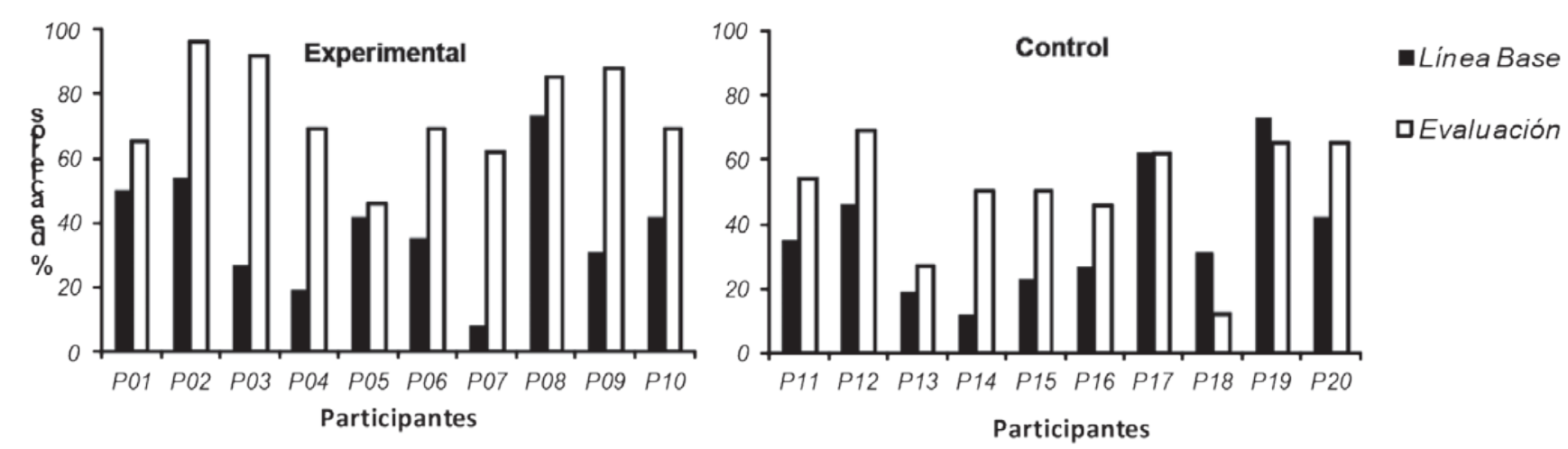

Figura 1. Porcentaje de aciertos obtenido en Línea Base y la Evaluación por los participantes de los grupos experimental y control. 
el porcentaje de aciertos logrado por cada uno de ellos. Las barras negras representan la LB, y las blancas, la evaluación. Como puede observarse, la mayoría de los participantes del grupo experimental logró un aumento de más del 30\% de la LB a la evaluación, lo que no ocurrió con el grupo control (con excepción del P14).

En la Figura 2 puede observarse el porcentaje de respuestas correctas del grupo experimental. La mayoría obtuvo un puntaje superior al 70\% en los dos ejercicios. Al menos la mitad logró un mejor desempeño en el segundo ejercicio que en el primero, lo que se evidencia en el menor número de sesiones que requirieron para lograr el 100\% de aciertos. Aunque algunos participantes tuvieron su peor desempeño en el ejercicio 2, dado que a pesar de que el entrenamiento era correctivo, en ocasiones argumentaron que "ya no se les ocurría" cómo corregir su ejercicio y preferían dejar sus respuestas como las tenían.
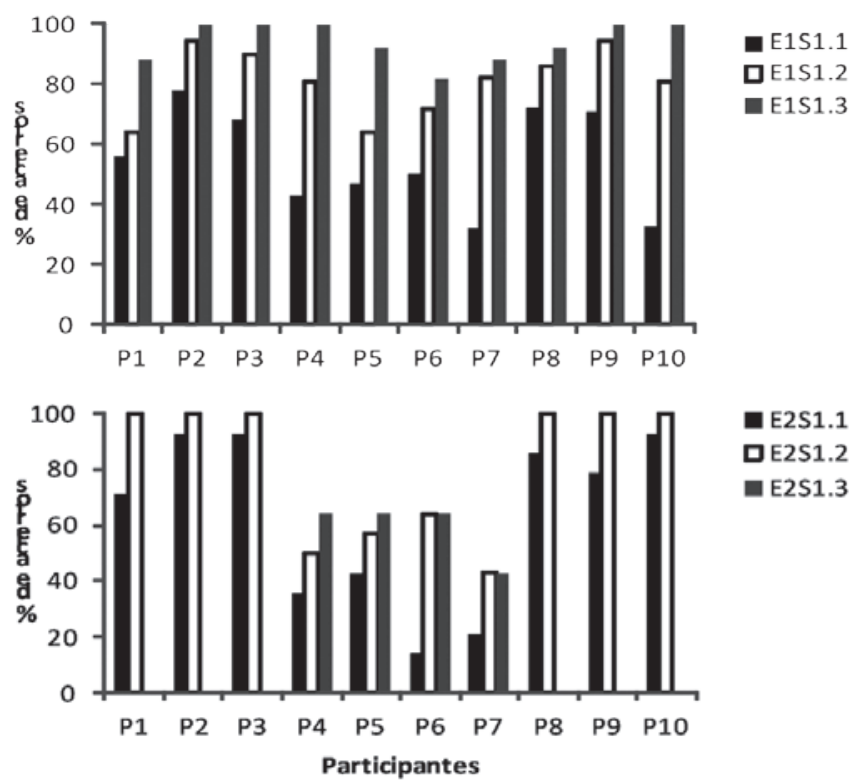

Resultados relativos a la extensión, pertinencia, congruencia y nivel funcional del anteproyecto elaborado

Con respecto a la extensión, en número de palabras del anteproyecto elaborado, así como a la pertinencia de los términos técnicos empleados en la LB y en la evaluación por los participantes del grupo experimental (véase Tabla 3 ), cuatro de los diez participantes aumentaron la extensión en 200 palabras o más, de la LB a la evaluación. Y en lo que se refiere a si emplearon o no los términos de manera pertinente para fundamentar la pregunta de investigación propuesta en su anteproyecto, entendiendo la pertinencia como el uso de tales términos en el mismo sentido en que se utilizaban en los artículos previamente leídos, cinco de los diez participantes del grupo experimental utilizaron los términos técnicos de manera adecuada en la evaluación, a diferencia de la LB en la que no los habían empleado.

Figura 2. Porcentaje de respuestas correctas obtenido en las tres sesiones de entrenamiento del grupo experimental. Figuras de la parte superior, resultados de las sesiones del ejercicio 1; figuras de la parte inferior, sesiones del ejercicio 2. Claves: E1S1.1= Ejercicio 1, Sesión 1, Exposición 1, etc.; E2S1.1= Ejercicio 2, Sesión 1, Exposición 1, etc. 
Tabla 3.

No. de palabras empleadas para elaborar las preguntas de investigación, y Congruencia, Pertinencia y Nivel funcional en que fueron escritas (E=Extrasituacional; I=Intrasituacional; $\mathrm{X}=$ Ausencia de respuesta*)

Grupo experimental

\begin{tabular}{|c|c|c|c|c|c|c|}
\hline Participante & Condición & $\begin{array}{l}\text { No. } \\
\text { palabras }\end{array}$ & de & Pertinencia & Congruencia & $\begin{array}{l}\text { Nivel } \\
\text { funcional }\end{array}$ \\
\hline \multirow{2}{*}{ P1 } & $\overline{L B}$ & 380 & & No & $\overline{\mathrm{Si}}$ & $\bar{E}$ \\
\hline & Ev & 647 & & $\mathrm{Si}$ & $\mathrm{Si}$ & E \\
\hline \multirow[t]{2}{*}{ P2 } & LB & 552 & & No & $\mathrm{Si}$ & I \\
\hline & Ev & 816 & & $\mathrm{Si}$ & $\mathrm{Si}$ & E \\
\hline \multirow[t]{2}{*}{ P3 } & LB & 27 & & No & $\mathrm{Si}$ & $\mathrm{X}$ \\
\hline & Ev & 1002 & & $\mathrm{Si}$ & $\mathrm{Si}$ & E \\
\hline \multirow[t]{2}{*}{ P4 } & LB & 310 & & No & $\mathrm{Si}$ & X \\
\hline & Ev & 390 & & No & $\mathrm{Si}$ & X \\
\hline \multirow[t]{2}{*}{ P5 } & LB & 380 & & No & $\mathrm{Si}$ & I \\
\hline & Ev & 369 & & No & $\mathrm{Si}$ & I \\
\hline \multirow[t]{2}{*}{ P6 } & LB & 140 & & No & No & I \\
\hline & Ev & 305 & & No & No & I \\
\hline \multirow[t]{2}{*}{ P07 } & LB & 269 & & No & No & I \\
\hline & Ev & 271 & & No & $\mathrm{Si}$ & I \\
\hline \multirow[t]{2}{*}{ P8 } & LB & 388 & & $\mathrm{Si}$ & $\mathrm{Si}$ & E \\
\hline & Ev & 435 & & $\mathrm{Si}$ & $\mathrm{Si}$ & E \\
\hline \multirow[t]{2}{*}{ P9 } & LB & 410 & & No & $\mathrm{Si}$ & I \\
\hline & Ev & 571 & & $\mathrm{Si}$ & $\mathrm{Si}$ & E \\
\hline \multirow[t]{2}{*}{ P10 } & LB & 352 & & No & $\mathrm{Si}$ & I \\
\hline & Ev & 1974 & & $\mathrm{Si}$ & $\mathrm{Si}$ & E \\
\hline
\end{tabular}

Grupo control

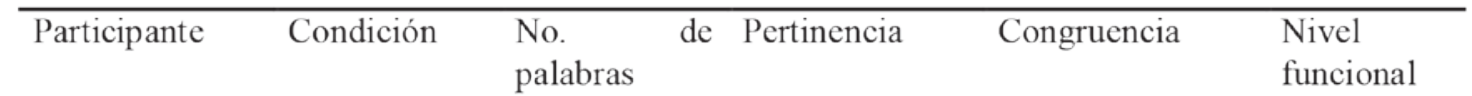

\begin{tabular}{|c|c|c|c|c|c|}
\hline \multirow[t]{2}{*}{ P11 } & LB & 219 & No & $\mathrm{Si}$ & I \\
\hline & Ev & 140 & No & $\mathrm{Si}$ & I \\
\hline \multirow[t]{2}{*}{ P12 } & LB & 384 & $\mathrm{Si}$ & $\mathrm{Si}$ & E \\
\hline & Ev & 846 & $\mathrm{Si}$ & $\mathrm{Si}$ & E \\
\hline \multirow[t]{2}{*}{ P13 } & LB & 357 & No & No & X \\
\hline & Ev & 585 & No & No & I \\
\hline \multirow[t]{2}{*}{ P14 } & LB & 118 & No & $\mathrm{Si}$ & X \\
\hline & Ev & 263 & No & $\mathrm{Si}$ & I \\
\hline \multirow{2}{*}{ P15 } & LB & 183 & No & No & I \\
\hline & Ev & 467 & No & $\mathrm{Si}$ & I \\
\hline \multirow[t]{2}{*}{ P16 } & LB & 323 & No & $\mathrm{Si}$ & $X$ \\
\hline & Ev & 233 & No & $\mathrm{Si}$ & I \\
\hline \multirow[t]{2}{*}{ P17 } & LB & 207 & No & $\mathrm{Si}$ & I \\
\hline & Ev & 209 & No & $\mathrm{Si}$ & I \\
\hline \multirow[t]{2}{*}{ P18 } & LB & 246 & No & No & I \\
\hline & Ev & 209 & No & No & I \\
\hline \multirow[t]{2}{*}{ P19 } & LB & 445 & $\mathrm{Si}$ & $\mathrm{Si}$ & I \\
\hline & Ev & 406 & $\mathrm{Si}$ & $\mathrm{Si}$ & I \\
\hline \multirow[t]{2}{*}{ P20 } & LB & 196 & No & No & I \\
\hline & Ev & 493 & No & $\mathrm{Si}$ & I \\
\hline
\end{tabular}

Nota: * Cuando la pregunta elaborada no tenía relación con los artículos leídos se consideraba como no pertinente y se evaluaba como ausencia de respuesta. 
Los participantes del grupo experimental que elaboraron las propuestas más extensas fueron, en general, los que lograron los niveles de desempeño más complejos (extrasituacionales). En cuanto al desempeño del grupo control, únicamente tres participantes mostraron un aumento de más de 200 palabras de la LB a la evaluación, y sólo cuatro utilizaron los términos técnicos de manera pertinente, aunque en tal caso ello no se relacionó con desempeños en niveles extrasituacionales, con excepción de dos casos, aunque ello ocurrió desde la LB.

En cuanto a la congruencia, que implicaba que los argumentos empleados para fundamentar la pregunta de investigación elaborada mostraran correspondencia lógica con dicha pregunta, se encontró que nueve participantes del grupo experimental y ocho del control formularon propuestas congruentes en la evaluación (véase Tabla 3).

En lo relativo al nivel funcional en el que escribieron y fundamentaron su pregunta de investigación, del grupo experimental seis de los diez participantes la propusieron en un nivel extrasituacional en la evaluación. Destacó el hecho de que los que lograron el mayor porcentaje de respuestas correctas en el ejercicio 2 y en la evaluación, mostraron desempeños en un nivel extrasituacional en esta última condición, mientras que los que obtuvieron los puntajes más bajos formularon sus propuestas en un nivel intrasituacional. Y en el grupo control sólo escribió su pregunta de investigación en el nivel extrasituacional un participante, quien ya lo había hecho desde la LB. El resto solo logró desempeños en un nivel intrasituacional o mostró ausencia de respuesta (véase Tabla 3), lo que implicaba que la pregunta propuesta no tenía ninguna relación con los artículos leídos.

Como puede observarse en la Tabla 3, dos participantes del grupo experimental y uno del control ya se comportaban en niveles extrasituacionales desde la LB, pero se decidió dejarlos como parte del estudio con el objeto de explorar si ese nivel de desempeño se mantenía en la evaluación en ambos grupos (tanto en los que se expondrían a entrenamiento como en los que no), si se modificaba la extensión de sus propuestas, o si cambiaba la pertinencia del uso de términos técnicos o la congruencia de la propuesta elaborada. Se observó que los tres mejoraron su desempeño en todos los aspectos medidos, de la LB a la evaluación, incluyendo el del grupo control que no se expuso a entrenamiento, lo que no ocurrió con el resto de los participantes de dicho grupo. Es importante mencionar que la comparación de los datos recabados, por el género de los participantes, no mostró diferencias en el desempeño.

\section{DISCUSIÓN}

Los resultados mostraron que el entrenamiento correctivo al que se expuso a los participantes promovió la elaboración y justificación de preguntas de investigación en niveles extrasituacionales. A diferencia de lo encontrado en el estudio de Padilla et al. (2009), en el que los sujetos obtuvieron puntajes bajos en los dos ejercicios del entrenamiento, en el presente se observaron ejecuciones altas en ambos, lo cual pareció favorecer el desempeño en la evaluación.

Un dato que se destaca es la correspondencia observada entre la ejecución lograda en el ejercicio 2 y el nivel en que se elaboraron las preguntas y su fundamentación, dado que un bajo porcentaje de aciertos en este se relacionó con un desempeño en un nivel intrasituacional y un alto puntaje con una ejecución en un nivel extrasituacional. Al parecer, esto demuestra que se logró el objetivo del entrenamiento de auspiciar desempeños en niveles extrasituacionales.

Este hallazgo concuerda con lo propuesto por Ribes y López (1985) respecto de que los niveles implican complejidades diferenciales y crecientes. Es decir, las grandes diferencias observadas en el desempeño de los participantes del grupo experimental en las sesiones de entrenamiento de los ejercicios 1 y 2 pueden explicarse en términos de la complejidad diferencial del comportamiento requerido, ya que resolver el ejercicio 1, que promovía ejecuciones en niveles intrasituacionales, solo implicaba identificar elementos de los artículos leídos, por lo que no es sorprendente que en dicho ejercicio la gran mayoría haya logrado ejecuciones con aciertos entre el $90 \%$ y $100 \%$, pero en el ejercicio 2 (que tenía como objetivo promover desempeños en niveles extrasituacionales) la ejecución de cuatro de los diez participantes haya sido baja.

$Y$ es que dicho entrenamiento, a diferencia del anterior, requería que los participantes no repitieran lo leído, sino que a partir de los artículos revisados identificaran que lo que articula y le da coherencia a un artículo científico es la pregunta de investigación, por lo que debían realizar actividades que implicaban analizar de manera crítica lo propuesto por los autores de los artículos revisados, lo cual requería el ejercicio de habilidades de elaboración, formulación, fundamentación, interpretación de evidencia, análisis crítico de lo leído y elaboración de una pregunta de investigación novedosa derivada de los artículos revisados; habilidades que demostraron no ser capaces de desplegar. Este hallazgo coincide con lo encontrado por Pacheco, Carranza, Silva, Flores y Morales (2005), quienes evaluaron el desempeño de estudiantes de psicología en tareas de identificación y elaboración de términos de la psicología experimental, y 
observaron porcentajes de respuestas correctas más altos en identificación que en elaboración.

Los resultados obtenidos por los cuatro participantes ya mencionados en el presente estudio parecen demostrar la incapacidad de estos para comportarse extrasituacionalmente a pesar del entrenamiento correctivo, lo cual, como ya se señaló, es consistente con el supuesto de la diferencia funcional existente entre los criterios de logro para realizar ambos tipos de ejercicios (el 1 y el 2 de este trabajo) y la complejidad diferencial entre ellos (Ribes \& López, 1985). Dichos datos concuerdan con lo observado por otros autores respecto de que el nivel funcional en el que se estructura una competencia verbal modula la probabilidad de que un individuo se comporte en ese mismo nivel cuando se expone a situaciones novedosas (Mares, Ribes, \& Rueda, 1993; Mares, Rueda, Plancarte, \& Guevara, 1997).

Se considera que esa gran diferencia observada en el desempeño de algunos participantes, del ejercicio 1 al 2, tal vez podría subsanarse aumentando el número de sesiones del entrenamiento correctivo, lo que implicaría incrementar la historia de referencialidad de los participantes al exponerlos a más artículos del área. Podría cambiarse el criterio de logro de uno de exposición (como se ha venido haciendo) a uno de ejecución. Eso es algo que habría que probar en estudios futuros.

El que todos los participantes del grupo experimental hayan aumentado su puntaje de la LB a la evaluación (36\% en promedio), mientras que los del grupo control sólo lo hayan hecho en un $13 \%$ en promedio, parece evidenciar la efectividad del entrenamiento correctivo empleado. El hecho de que el grupo control también haya mejorado su puntaje parece indicar que la sola lectura de los artículos tuvo un efecto sobre este aspecto concreto, aparentemente por el incremento de su historia de referencialidad con la temática de los textos.

En lo referente a la pertinencia en el uso de los términos empleados para fundamentar la pregunta de investigación elaborada, el que cinco de los participantes del grupo experimental, luego de exponerse al entrenamiento, empezaran a emplearlos de manera pertinente (uno ya lo hacía desde la LB) y que ello se relacionara en todos los casos con desempeños en niveles extrasituacionales (mientras que en el control no ocurrió así), parece indicar que el solo hecho de leer los artículos, sin exponerse a un entrenamiento en la identificación y elaboración de elementos de éstos, no favorece la identificación de argumentos que permitan fundamentar una pregunta de investigación de manera adecuada.

Tal hallazgo ya se había observado en un estudio previo en el que se analizaron los efectos del dominio diferencial de los supuestos teóricos y metodológicos de una teoría sobre la elaboración y justificación de preguntas de investigación. Se encontró que a mayor entrenamiento en los elementos de una teoría (definiciones, ejemplos y/o experimentos), mayor era la cantidad de participantes que empleaban términos técnicos (y de manera pertinente) de la teoría entrenada, para elaborar y justificar una pregunta de investigación (Padilla et al., 2010).

En cuanto a la congruencia, aparentemente el tratamiento al que se expuso a los participantes no afectó dicho aspecto, dado que no se observaron diferencias relevantes entre ambos grupos. En lo que se refiere a la extensión de las propuestas elaboradas, se encontró lo esperado: los participantes del grupo experimental la aumentaron sustancialmente de la LB a la evaluación, dado que, al parecer, el haberse expuesto al entrenamiento les permitió identificar los criterios pertinentes para elaborar y fundamentar una pregunta de investigación de manera adecuada, empleando una mayor cantidad de evidencias y planteamientos derivados que los usados en la LB. Este efecto no fue tan marcado en el grupo control (a pesar de haber leído los mismos artículos que el experimental), ya que incluso tres participantes de este último grupo disminuyeron la extensión de sus propuestas, y en promedio, el aumento de los que sí las ampliaron fue bastante menor con respecto a lo observado en el grupo experimental.

Se observó que a pesar del entrenamiento correctivo los resultados del presente estudio fueron similares a los del estudio de Padilla et al. (2009); una diferencia fundamental entre ambos estudios fue la población empleada, ya que en el estudio de Padilla et al. (2009) los participantes eran de posgrado, mientras que los del presente eran de licenciatura. Ello es relevante dado que los estudiantes de posgrado sí poseen una historia de contacto referencial con textos técnicos, mientras que los de licenciatura no. Y a la luz de esa diferencia los datos del presente estudio cobran otra relevancia.

Aunque el entrenamiento de este trabajo era correctivo, en algunas sesiones cuatro de los participantes no corrigieron sus escritos o no corrigieron lo crítico, sino sólo aspectos superficiales, lo que ya se ha encontrado en estudios previos en los que se ha observado que cuando se retroalimenta a escritores novatos, lo que estos hacen es corregir lo superficial, como la ortografía y la gramática, pero no lo fundamental, como la estructura y su contenido (Hyland, 2003).

El bajo desempeño observado en algunos participantes, en el ejercicio 2, demuestra que los estudiantes de pregrado tienen dificultades para escribir, y si a ello se agrega el requisito de escribir técnicamente, como lo requería este ejercicio, el desempeño es peor, como lo muestran los datos. Esto constituye una alerta sobre la importancia que tiene 
el exponer a los estudiantes de pregrado en general, y a los de psicología en particular (dado que es la población en la que más se han analizado las dificultades lectoescritoras), a un entrenamiento explícito en el manejo del lenguaje de su área disciplinar, lo que implica ser capaces de leer, escribir, escuchar y hablar en los términos técnicos de la disciplina en la que los estudiantes estén siendo formados (Moreno, 2005).

Las habilidades lectoescritoras de textos técnicos se aprenden mediante el ejercicio supervisado, el modelamiento, la evaluación-corrección y la explicitación de los criterios pedagógicos y disciplinarios a cumplir (Pacheco, 2010). Sin embargo, en un estudio de Pacheco (2008) se encontró que los docentes no planeaban de manera explícita el entrenamiento de tales habilidades ni seguían estrategias estructuradas para evaluar si sus estudiantes eran capaces de ejercitar tales habilidades.

Tal situación ya se había identificado al analizar los planes curriculares de la carrera a la que pertenecían los participantes en el estudio previamente citado (Aguilera et al., 2006); pero a pesar de ello, en la propuesta posterior al estudio no se contempló implementar un programa orientado a auspiciar habilidades en ese sentido (Pacheco, 2010). No obstante, en otros países, como Colombia, sí existen algunos programas cuyo objetivo es el desarrollo de habilidades de investigación en los que se entrena, entre otros aspectos, en habilidades de lectura, análisis de textos y generación de escritos (Guerrero, 2007).

$Y$ dado que se espera que los universitarios dominen el lenguaje técnico de su disciplina, evaluar habilidades lectoescritoras en esta población implica tener en cuenta la experiencia o dominio que ellos tienen de dicho lenguaje (Johnstone, Ashbaugh, \& Warfield, 2002), pero lo que se ha observado es que, en general, este es limitado (Fonseca \& Padilla, 2012; Padilla et al., 2010).

En el presente estudio seis de los diez participantes lograron excelentes desempeños después de exponerse al entrenamiento, y fueron capaces de elaborar y fundamentar adecuadamente sus preguntas de investigación empleando para ello el lenguaje técnico de los artículos recién leídos. Sin embargo, para cuatro de ellos eso fue imposible, a pesar del entrenamiento correctivo al que se les expuso; al parecer, se les dificultó interactuar con el lenguaje técnico disciplinar, lo que les impidió ajustarse a los criterios de logro estipulados en el entrenamiento. De manera anecdótica se tiene el registro de que tales participantes reportaban que la tarea les parecía compleja, difícil y agotadora. Dicho dato parece indicar que los participantes no estaban motivados para realizar las actividades que implicaba el estudio, lo que algunos autores denominan como una actitud de "poca orientación al logro", en lo que a resultados académicos se refiere (Mújica, Guido, \& Gutiérrez, 2012; Valdés, Sánchez, \& Yáñez, 2013).

Pero el dominio del lenguaje científico se puede lograr fácilmente con un entrenamiento adecuado, ya que Kelly y Chen (1999) encontraron que luego de exponer a estudiantes de secundaria a un procedimiento en el que se les explicitaba claramente el objetivo a lograr con esa actividad y se les proporcionaba una guía que tenía como meta enseñarles a escribir de manera ordenada, se observó que estos escribían de manera más sistemática y utilizaban un mayor número de términos científicos correspondientes a la teoría a la que se les había expuesto. Y si ello se logró con estudiantes de secundaria, con participantes de niveles superiores, como psicólogos en formación, los resultados podrían ser más prometedores, como lo han demostrado los estudios aquí reseñados. Por lo tanto, se sugiere seguir trabajando en la identificación de las condiciones que promuevan la elaboración y fundamentación de preguntas de investigación escritas en niveles extrasituacionales, con todo lo que ello implica. Dicho análisis debe realizarse con estudiantes de diferentes áreas disciplinares, con el objeto de verificar la posible replicación de los hallazgos obtenidos hasta ahora con psicólogos en formación.

Finalmente, también se considera importante analizar los otros elementos implicados en las interacciones escritoras, como son la historia de contacto con los referentes respecto de lo que se va a escribir, las características del material a partir del cual se va a escribir, las habilidades lingüísticas de que dispone el participante (como hablante, escucha y lector) y sus características disposicionales (como su estado de ánimo, motivación hacia la tarea, etc.), entre otros aspectos (Pacheco, 2010), para poder contar con más elementos que permitan planear estrategias integrales de entrenamiento en habilidades lectoescritoras de materiales técnicos.

\section{REFERENCIAS}

Aguilera, G., Alcaraz, R., Cabrera, R., Cabrera, N., Galicia, X., Hickman, H., Mares, G., Mejía, O., Quintanar, F. \& Robles, S. (2006). Comisión de evaluación del plan de estudios vigente (Documento interno). México: FES-Iztacala.

Arroyo, R., Canales, C., Morales, G., Silva, H. \& Carpio, C. (2007). Programa de investigación para el análisis funcional del ajuste lector. Acta Colombiana de Psicología, 10(2), 31-39.

García, F. C., García, Á., Berbén, A. B. G., Pichardo, M. C. \& Justicia, F. (2014). The Effects of Question-Generation Training on Metacognitive Knowledge, Self-Regulation and Learning Approaches in Science. Psicothema, 26(3), 385-390. 
Cassany, D. (2006). Taller de textos. Leer, escribir y comentar en el aula. España: Paidós.

De la Fuente, J., Justicia, F., Casanova, P. F. \& Trianes, M. V. (2005). Perceptions about the Construction of Academic and Professional Competencies in Psychologists. Electronic Journal of Research in Educational Psychology, 3(1), 3-34.

Fonseca, L. C. \& Padilla, M. A. (2012). Effects of Differential Exposure to Terms in Ordinary Language and Technical Terms on the Explanation of Psychological Phenomena. Cartel presentado en la 2012 Theory and Philosophy Conference, Santa Fe, NM, EE.UU.

González-Torres, M. L., Padilla, M. A. \& Tamayo, J. (2011). Effects of the Degree of Specificity of the Achievement Criterion on the Informal Questions Elaboration. International Journal of Hispanic Psychology, 3(2), 171-183.

Guerrero, M. E. (2007). Formación de habilidades para la investigación desde el pregrado. Acta Colombiana de Psicología, 10 (2), 190-192.

Hyland, F. (2003). Focusing on Form: Student Engagement with Teacher Feedback. System, 31(2), 217-230.

Irigoyen, J., Jiménez, M. \& Acuña, K. (2004). Evaluación del ejercicio instruccional en la enseñanza universitaria. Enseñanza e Investigación en Psicología, 9(2), 293-302.

Johnstone, K., Asbaugh, H. \& Warfield, T. (2002). Effects of Repeated Practice and Contextual-Writing Experiences on College Students Writing Skills. Journal of Education Psychology, 94(2), 305-315.

Kelly, G. \& Chen, C. (1999). The Sound of Music: Constructing Science as Sociocultural Practices through Oral and Writing Discourse. Journal of Research in Science Teaching, 36(8), 883-915.

Keys, C. W., Hand, B., Prain, V. \& Collins, S. (1999). Using the Science Writing Heuristic as a Tool for Learning from Laboratory Investigations in Secondary Science. Journal of Research in Science Teaching, 36(10), 1065-1084.

Lyster, R. \& Ranta, L. (1997). Corrective Feedback and Learner Uptake: Negotiation of Form in Communicative Classrooms. Studies in Second Language Acquisition, 19, 37-66.

Madariaga, J. M. \& Martínez, E. (2010). La enseñanza de estrategias de comprensión y metacomprensión lectora. Un programa implementado por el profesorado. Anales de Psicología, 26(1), 112-122.

Mares, G., Ribes, E. \& Rueda, E. (1993). El nivel de funcionalidad en lectura y su efecto sobre la transferencia de lo leído. Revista Sonorense de Psicología, 7(1), 32-43.

Mares, G., Rueda P, E., Plancarte, P. \& Guevara, Y. (1997). Conducta referencial no entrenada: El papel que juega el nivel funcional de entrenamiento en la generalización. Acta Comportamentalia, 5(2), 199-219.

Montealegre, R. \& Forero, L. A. (2006). Desarrollo de la lectoescritura: Adquisición y dominio. Acta Colombiana de Psicología, 9(1), 25-40.
Moreno, M. (2005). Potenciar la educación. Un curriculum transversal de formación para la investigación. Revista Electrónica Iberoamericana sobre Calidad, Eficiencia y Cambio en Educación, 3, 520-540.

Mújica S. A., Guido, P. G. \& Gutiérrez, R. R. M. (2012). Factores motivacionales y de capital cultural que inciden en el comportamiento lector en estudiantes mexicanos de nivel medio superior de diferente estrato social. Acta Colombiana de Psicología, 15(1), 21-31.

Oduber, O. I. G. \& Matos, R. J. M. (2009). Retroalimentación oral correctiva en el aula de inglés como lengua extranjera (ILE). Laurus, 15(29), 339-367.

Pacheco, V., Carranza, N., Silva, H., Flores, C. \& Morales, G. (2005). Evaluación del aprendizaje de la práctica científica en psicología. En: C. Carpio \& J. Irigoyen. Psicología y Educación. México, UNAM.

Pacheco, V., Ramírez, L., Palestina, L. \& Salazar, M. (2007). Una aproximación al análisis funcional de la relación entre las conductas de leer y escribir en estudiantes de psicología. En J. J. Irigoyen, M. Jiménez y K. Acuña (Eds.), Enseñanza, aprendizaje y evaluación. Una aproximación a la pedagogía de las ciencias (pp. 247-275). México: UniSon.

Pacheco, V. (2008). Generación de conocimiento y habilidades escritoras: Un análisis de la producción de textos en universitarios. Tesis doctoral, Universidad Nacional Autónoma de México, México, D.F.

Pacheco, V. (2010). ¿Se enseña a escribir a los universitarios? Análisis y propuesta desde la teoría de la conducta. México: UNAM FES Iztacala.

Padilla, M. A., Solórzano, W. \& Pacheco, V. (2009). Effects of the Degree of Specificity of the Achievement Criterion on the Informal Questions Elaboration. Electronic Journal of Research in Educational Psychology, 7(17), 77-102.

Padilla, M. A., Suro, A. L. \& Tamayo, J. (2010). Efectos de la exposición diferencial a los supuestos de una teoría en la elaboración de preguntas de investigación. Revista Mexicana de Psicología, 27(2), 247-256.

Padilla, M. A., Tamayo, J. \& González, M. L. (2010). Análisis de la posible relación entre la elaboración de preguntas informales y de investigación. IPyE: Psicología y Educación, 4(7), 1-21.

Paradiso, J. C. (1996). Comprensión de textos expositivos: Estrategias para el aula. Anales de psicología, 12(2), 167-177.

Ribes, E. \& López, F. (1985). Teoría de la conducta. Un análisis de campo y paramétrico. México: Trillas.

Ribes, E. \& Serrano, M. (2006). Efectos de tres tipos de preentrenamiento en la adquisición y transferencia de una tarea de igualación de la muestra. Acta Comportamentalia, 14(2), 145-169.

Ribes, E., Vargas, I., Luna, D. \& Martínez, C. (2009). Adquisición y transferencia de una discriminación condicional en una secuencia de cinco criterios distintos de ajuste funcional. Acta Comportamentalia: Revista Latina de Análisis del Comportamiento, 17(3). 
Sánchez, P. R. (2004). Enseñar a investigar. Una didáctica nueva de la investigación en ciencias sociales y humanas. México: Plaza y Valdés.

Tamayo, J., Padilla, M. A. \& González-Torres, M. L. (2009). Efectos de criterios de logro diferenciales en la elaboración de preguntas informales, en estudiantes de preparatoria, licenciatura y posgrado. Acta Colombiana de Psicología, 12(1), 27-39.
Valdés C. Á. A., Sánchez E. P. A. \& Yáñez Q. A. I. (2013). Perfiles de estudiantes mexicanos con aptitudes intelectuales sobresalientes. Acta Colombiana de Psicología, 16(1), 25-33.

Viniegra, L. (2002). Un acercamiento a la crítica. Educación y Crítica: El proceso de elaboración del conocimiento. México: Paidós. 